Arch. Tierz., Dummerstorf 50 (2007) 2, 165-173

Aus dem Institut für Tierzucht und Haustiergenetik der Justus-Liebig-Universität Gießen

STEFFEN HOY, CARMEN WEIRICH und STEPHANIE KNOOP

\title{
Körpermasseentwicklung von Absetzferkeln bei unterschiedlichen Tier-Fressplatz-Verhältnissen
}

\begin{abstract}
Title of the paper: Investigations on live weight development of weaner pigs fed with different pigletfeeding place ratios

Investigations with a total of 656 weaner pigs in 14 rounds fed either with a pig-feeding place-relation of $1: 1$ (Piglet-Feeder, Tipp-Feeder) or 5 to $8: 1$ (tube wet feeders) under standardized conditions of a research station in groups of 20 each or under farm conditions in groups of 32 each have shown that piglets with a pig-feeding place-relation had higher daily gain by $25 \mathrm{~g}$ up to $48 \mathrm{~g}$ during the raising period on average of the groups compared with tube feeder groups. The live weight development of weaner pigs in groups with a piglet-feeding place-ratio of $1: 1$ was more homogeneous compared with groups with a wide weanling-feeding place-ratio (5 to $8: 1$ ) characterized by lower coefficients of variation for the daily gain by $5.8 \%$ up to $13.2 \%$.
\end{abstract}

Key Words: weaner pig, live weight development, piglet-feeding place-ratio, feed intake, Piglet-Feeder, TippFeeder, tube wet feeder

\section{Zusammenfassung}

Untersuchungen in 14 Durchgängen an insgesamt 656 Absetzferkeln, die entweder mit einem Tier-FressplatzVerhältnis von 1 : 1 (am Ferkelfeeder bzw. Tipp-Feeder) oder 5 bzw. 8 : 1 (Rohrbreiautomaten) unter den standardisierten Bedingungen einer Forschungsstation in Gruppen zu je 20 Tieren oder in einem Praxisbetrieb in Gruppen zu je 32 Tieren gefüttert wurden, ergaben 25 g bis 48 g höhere tägliche Zunahmen im Mittel der Gruppen während der Aufzuchtperiode, in denen jedes Ferkel einen Fressplatz zur Verfügung hatte, im Vergleich zu den an Breiautomaten mit einer Tier-Fressplatz-Relation von 5 bis $8: 1$ gefütterten Stallgefährten. Die Lebendmasseentwicklung der Ferkel in den Gruppen mit einem 1 : 1-Verhältnis von Tieren zu Fressplätzen war deutlich ausgeglichener gegenüber Ferkelgruppen mit einem weiten Tier-Fressplatz-Verhältnis $(5$ bis $8: 1)$, was durch 5,8 \% bis 13,2 \% geringere Variationskoeffizienten für die täglichen Zunahmen dokumentiert wurde.

Schlüsselwörter: Absetzferkel, Lebendmasseentwicklung, Tier-Fressplatz-Verhältnis, Futteraufnahme, Ferkelfeeder, Tipp-Feeder, Rohrbreiautomat

\section{Einleitung}

Beim Absetzen der Ferkel von der Sau treffen viele belastende Faktoren zusammen (HELD und MENDL, 2001). Bis zum Absetzen trinken die Ferkel zeitlich synchron etwa im Stundentakt ein flüssiges Futtermittel (Muttermilch), wobei jedes Ferkel einen festen Fressplatz (Zitzenposition) hat, was einem Tier-Fressplatz-Verhältnis von $1: 1$ entspricht (HOY und MEHLHORN, 1989; HOY und PUPPE, 1992; PUPPE et al., 1993). Festes Beifutter wird zunächst nur in geringen Mengen (in Abhängigkeit von der Länge der Säugezeit, der Milchleistung der Mutter, der Qualität des Beifutters u.a.) aufgenommen (BROOKS und TSOURGIANNIS, 2003). Die Nahrung wird zur freien Aufnahme in größeren Gruppen bei einem weiten Tier-Fressplatz-Verhältnis (häufig $8: 1$ oder sogar noch mehr) angeboten (MADEC et al., 2003; HOY, 2004, 2005). Vor allem für die kleineren Ferkel bedeutet dies eine starke Belastung, wenn sie sich mit 
sieben, acht oder noch mehr fremden Ferkeln um einen Platz am Futterautomaten auseinandersetzen müssen (SCHÄFER, 1999). Die Ferkel werden von der Mutter getrennt und in eine fremde Umgebung umgestallt, wobei nach der Gruppenzusammenstellung Rangkämpfe entstehen (MORMEDE und HAY, 2003). Die Situation kann dann eskalieren, wenn Ferkel verschiedener Herkünfte zusammen aufgestallt werden und Postweaning Multisystemic Wasting Syndrome (PMWS) durch porcine Circovirus-Infektion in Verbindung mit PRRS auftritt (DONE, 2001; MADEC et al., 2003). Die Ferkel reagieren mit Verlusten, verringerten Zunahmen und Kümmern. Neben der räumlichen Trennung der Absetzferkel von der Sauenherde und der isolierten Aufzucht im Interesse der Gesunderhaltung der Tiere hat eine tiergerechte Fütterungstechnik, die mehrmals am Tag kleine Portionen zur rationierten Futteraufnahme bei einem Tier-Fressplatz-Verhältnis von annähernd $1: 1$ zumindest in der absetznahen Periode anbietet, eine große Bedeutung. Das Ziel der vorliegenden Untersuchungen bestand darin, die Ausgeglichenheit der Körpermasseentwicklung von Absetzferkeln bei einem Tier-Fressplatz-Verhältnis von $1: 1$ an neuen Fütterungstechniken (Ferkelfeeder, Tipp-Feeder) im Vergleich zu einem Tier-FressplatzVerhältnis von 5 bzw. 8 : 1 bei herkömmlicher Technik (Breiautomat) zu untersuchen.

\section{Material und Methoden}

Die Untersuchungen fanden an 400 eingestallten Ferkeln in 10 Haltungsdurchgängen in zwei klimatisierten Stallabteilen der Lehr- und Forschungsstation Oberer Hardthof des Institutes für Tierzucht und Haustiergenetik sowie an 256 Ferkeln in 4 Wiederholungen in einem Praxisbetrieb statt (Tab. 1). In beiden Abteilen der Forschungsstation wurde je eine Bucht für 20 Ferkel $\left(0,30 \mathrm{~m}^{2} /\right.$ Tier $)$ mit Kunststoffrosten eingerichtet. In einem Abteil befand sich während der ersten fünf Durchgänge der Ferkelfeeder (KNOOP und HOY, 2005) und in den anderen fünf Haltungsdurchgängen der Tipp-Feeder (HOY, 2006). Beide Techniken besaßen einen Doppellängstrog (1,50 m lang), so dass bei einer gesamten Troglänge von $300 \mathrm{~cm}$ und einer Fressplatzbreite von $15 \mathrm{~cm}$ je Ferkel ein Tier-Fressplatz-Verhältnis von $1: 1$ realisiert wurde. Der Unterschied zwischen den beiden Techniken bestand darin, dass beim Ferkelfeeder in fünf Blöcken bis zu 18mal pro Tag sensorkontrolliert eine definierte Portion Futter auf Wasser in den Trog verabreicht wurde und diese Menge verzehrsabhängig gesteigert wurde (KNOOP und HOY, 2005). Am Tipp-Feeder konnten die Ferkel ständig durch Betätigen einer Dosierklappe kleine Mengen an Futter auf Wasser dosieren - entsprechend ihres Bedarfes (HOY, 2006). Als Vergleichstechnik diente in zehn Wiederholungen ein Breiautomat (AP-Swing - Fa. Agro Products) zur ad libitum-Fütterung mit einem Tier-Fressplatz-Verhältnis von 5 : 1. Jeweils 40 Absetzferkel pro Durchgang mit einem mittleren Absetzalter von 28,5 Tagen wurden nach Absetzgewicht, Geschlecht, Genetik (Landrasse, Edelschwein sowie Hybriden aus Anpaarung der genannten Mutterlinien mit Pietrain-Ebern) und Herkunftswurf paritätisch auf die beiden jeweils zu vergleichenden Gruppen (1: 1 vs 5 : 1) aufgeteilt. Die Raumtemperatur bei Einstallung betrug $28{ }^{\circ} \mathrm{C}$, zusätzlich waren in den ersten Tagen nach dem Absetzen Infrarotstrahler zur lokalen Heizung im Einsatz. Die Raumtemperatur wurde schrittweise bis zum Ende der etwa 5,5wöchigen Aufzuchtperiode abgesenkt. Die Ferkel wurden einzeln bei Einstallung sowie bei Ausstallung gewogen. Täglich wurde für beide Gruppen die Menge des industriell gefertigten Ferkelfutters (Ferkelstarterfutter: 20,0 \% Rohprotein, 8,5 \% Rohfett, 2,5 \% 
Rohfaser, 1,6 \% Lysin; Ferkelaufzuchtfutter ab 2./3. Woche nach dem Absetzen: 16,0 \% Rohprotein, 4,6 \% Rohfett, 4,5 \% Rohfaser, 1,35 \% Lysin) eingewogen.

Tabelle 1

Untersuchungsdesign (Design of investigations)

\begin{tabular}{c|c|c|c|c|c}
\hline $\begin{array}{c}\text { Durch- } \\
\text { gänge }\end{array}$ & Ort & Fütterungstechnik & TFV & $\begin{array}{c}\text { Anzahl Tiere/Durchgang } \\
\text { und Fütterungstechnik }\end{array}$ & $\begin{array}{c}\text { Anzahl Tiere } \\
\text { insgesamt }\end{array}$ \\
\hline $1-5$ & $\begin{array}{c}\text { Forschungs- } \\
\text { station }\end{array}$ & Ferkelfeeder & $1: 1$ & 20 & 100 \\
& Rohrbreiautomat & $5: 1$ & 20 & 100 \\
\hline $6-10$ & $\begin{array}{c}\text { Forschungs- } \\
\text { station }\end{array}$ & Tipp-Feeder & $1: 1$ & 20 & 100 \\
\hline $11-14$ & Rohrbreiautomat & $5: 1$ & 20 & 128 \\
& & Ferkelfeeder & $1: 1$ & 32 & 128
\end{tabular}

TFV = Tier-Fressplatz-Verhältnis

In den vier Wiederholungen im Praxisbetrieb wurden 256 Ferkel (Masthybriden aus Landrasse x Pietrain) gleichmäßig nach Lebendmasse und Geschlecht nach 21tägiger Säugezeit auf zwei Gruppen zu je 32 Ferkel aufgeteilt. In einer Gruppenbucht mit Kunststoffrostenboden $\left(0,30 \mathrm{~m}^{2} /\right.$ Tier $)$ befand sich der Ferkelfeeder mit einem TierFressplatzverhältnis von $1: 1$, der analog zur Forschungsstation bewirtschaftet wurde. Im zweiten Abteil war ein Rohrbreiautomat installiert, mit dem 32 Ferkel versorgt wurden, was einem Tier-Fressplatz-Verhältnis von 8 : 1 entsprach. Auch hierbei wurden sämtliche Ferkel einzeln bei Ein- und Ausstallung gewogen, so dass daraus die täglichen Zunahmen zu berechnen waren. Bedingt durch die um eine Woche kürzere Säugezeit bei diesen Tieren im Vergleich zur Forschungsstation war die Haltungsdauer dementsprechend um eine Woche länger. Das Ferkelfutter, das hinsichtlich seiner Zusammensetzung dem in der Forschungsstation entsprach, wurde am Ferkelfeeder sensorgesteuert verzehrsabhängig und am Rohrbreiautomaten ad libitum verabreicht. Von den insgesamt 656 eingestallten Ferkeln in den drei Untersuchungsreihen schieden 25 (= 3,8 \%) aus. Einige Tiere verendeten an Colienterotoxämie, andere wurden wegen Gelenkentzündungen oder Schwanzbeißen aus den Gruppen genommen - ohne Bezug zum jeweiligen Fütterungssystem.

Sämtliche Einzeltierdaten wurden mit Hilfe des Statistik-Programmpaketes SPSS for Windows 11.5 einer statistischen Bearbeitung unterzogen. Nach der Berechnung der statistischen Maßzahlen für die gesamte Stichprobe bzw. die Teilstichproben entsprechend der Untersuchungsansätze fand eine Prüfung der Mittelwertunterschiede bezüglich der täglichen Zunahmen mittels t-Test statt. Außerdem wurde eine univariate Varianzanalyse mit den fixen Effekten von Geschlecht und Rasse (nur Forschungsstation) sowie der Kovariable Lebendmasse bei Einstallung in den Aufzuchtstall durchgeführt.

\section{Ergebnisse}

Die Vergleichsuntersuchungen in 5 Durchgängen (200 Ferkel) auf der Forschungsstation unseres Institutes zeigten übereinstimmend, dass am Ferkelfeeder mit einem TierFressplatz-Verhältnis von $1: 1$ gegenüber Rohrbreiautomaten und einem Tier-Fress- 
platz-Verhältnis von 5 : 1 die täglichen Zunahmen bei nahezu identischer Absetzlebendmasse (9,36 kg bzw. 9,32 kg) im Mittel um 33 Gramm signifikant höher waren als am Breiautomat mit einer Tier-Fressplatz-Relation von 5 : 1: Ferkelfeeder 417 Gramm, Breiautomat 384 Gramm (Tab. 2). Darüber hinaus ließ sich zeigen, dass die Standardabweichung für die täglichen Zunahmen bei einem Fressplatz je Ferkel mit \pm $101 \mathrm{~g}$ versus $\pm 123 \mathrm{~g}$ bei einem Fütterungssystem mit 5 Tieren pro Futterplatz geringer ausfiel. Somit war der Variationskoeffizient als Maß für die Ausgeglichenheit der täglichen Zunahmen bei einem Tier-Fressplatz-Verhältnis von 1 : 1 deutlich niedriger (24,2 \%) als bei einem solchen von 5 : 1 (32,0 \%; Tab. 5).

\section{Tabelle 2}

Leistungen von Absetzferkeln bei einem Tier-Fressplatzverhältnis von $1: 1$ (Ferkelfeeder) bzw. 5 : 1 (Rohrbreiautomat) (insgesamt 5 Durchgänge mit 200 Ferkeln) (Performance of weaner pigs fed with a pigletfeeding place-ratio of $1: 1$ (Piglet Feeder) or $5: 1$ (tube wet feeder)(totally 5 rounds with 200 piglets))

\begin{tabular}{lcc}
\hline Parameter & Ferkelfeeder & Rohrbreiautomat \\
Tier-Fressplatz-Verhältnis & $1: 1$ & $5: 1$ \\
\hline Absetzmasse $(\mathrm{kg})(\mathrm{n}=100: 100)$ & $\mathrm{x} \pm \mathrm{s} \pm \mathrm{s}$ \\
Ausstallmasse $(\mathrm{kg})(\mathrm{n}=98: 94)$ & $24,8 \pm 4,4,1,1,2$ & $23,6 \pm 4,9$ \\
Haltungstage & $37,0 \pm 3,3$ & $37,2 \pm 3,3$ \\
Tägliche Zunahmen $(\mathrm{g})(\mathrm{n}=98: 94)$ & $417 \pm 101$ & $384 \pm 123 *$
\end{tabular}

$* \mathrm{p}<0,05$

\section{Tabelle 3}

Leistungen von Absetzferkeln bei einem Tier-Fressplatzverhältnis von 1 : 1 (Tipp-Feeder) bzw. 5 : 1 (Rohrbreiautomat) (insgesamt 5 Durchgänge mit 200 Ferkeln) (Performance of weaner pigs fed with a pigletfeeding place-ratio of $1: 1$ (Tipp-Feeder) or $5: 1$ (tube wet feeder) (totally 5 rounds with 200 piglets))

\begin{tabular}{lcc}
\hline Parameter & Tipp-Feeder & Rohrbreiautomat \\
Tier-Fressplatz-Verhältnis & $1: 1$ & $5: 1$ \\
\hline Absetzmasse $(\mathrm{kg})(\mathrm{n}=100: 100)$ & $\mathrm{x} \pm \mathrm{s} \pm \mathrm{s}$ \\
Ausstallmasse $(\mathrm{kg})(\mathrm{n}=96: 97)$ & $26,1 \pm 4,6$ & $8,70 \pm 1,7$ \\
Haltungstage & $37,8 \pm 5,4$ & $25,1 \pm 4,7$ \\
Tägliche Zunahmen $(\mathrm{g})(\mathrm{n}=96: 97)$ & $455 \pm 95$ & $37,9 \pm 5,4$ \\
\end{tabular}

In fünf Wiederholungen auf der Forschungsstation mit insgesamt 200 eingestallten Ferkeln am Tipp-Feeder vs Rohrbreiautomat (je 100 Tiere) betrug die Absetzmasse (= Einstallmasse in den Aufzuchtstall) identisch 8,7 kg (Tab. 3). Die Ferkel erreichten am Tipp-Feeder mit einem Tier-Fressplatz-Verhältnis von 1 : 1 im Mittel um 25 Gramm bessere tägliche Zunahmen und eine um ein Kilogramm höhere Körpermasse nach 38 Haltungstagen (Tier-Fressplatz-Verhältnis $1: 1=455$ g, Tier-Fressplatz-Verhältnis 5 : $1=430$ g) (Tab. 3). In allen fünf Durchgängen waren die Tageszunahmen am TippFeeder tendenziell höher, wobei in den letzten beiden Durchgängen der Vorteil 47 
bzw. 54 Gramm pro Tier und Tag im Vergleich zum Rohrbreiautomaten betrug. Auch in dieser Untersuchungsserie war die Standardabweichung für die täglichen Zunahmen während der Aufzucht bei einer Tier-Fressplatz-Relation von $1: 1$ geringer $( \pm 95 \mathrm{~g})$ als bei einem Tier-Fressplatz-Verhältnis von $5: 1$ ( $\pm 103 \mathrm{~g})$. Analog zu den ersten fünf Haltungsdurchgängen war somit der Variationskoeffizient für die täglichen Zunahmen am Tipp-Feeder mit 20,7 \% niedriger als am Rohrautomat, bei dem für 5 Tiere ein Fressplatz zur Verfügung stand (23,8 \%; Tab. 5). Die Ergebnisse der univariaten Varianzanalyse zeigten, dass weder das Geschlecht oder der Genotyp noch die Lebendmasse beim Absetzen die täglichen Zunahmen im Aufzuchtabschnitt signifikant beeinflussten. Die um die genannten Effekte bereinigten Daten für die täglichen Zunahmen (eine Absetzmasse von 8,7 kg im Modell unterstellt) waren nahezu identisch mit den Rohdaten (Tier-Fressplatz-Verhältnis von $1: 1=454$ g; TierFressplatz-Verhältnis von $5: 1=435 \mathrm{~g})$.

Tabelle 4

Leistungen bei einem Tier-Fressplatzverhältnis von $1: 1$ (Ferkelfeeder) bzw. $8: 1$ (Rohrbreiautomat) (insgesamt 4 Durchgänge mit 256 Ferkeln) (Performance of weaner pigs fed with a piglet-feeding place-ratio of $1: 1$ (Piglet Feeder) or $8: 1$ (tube wet feeder)(totally 4 rounds with 256 piglets))

\begin{tabular}{|c|c|c|}
\hline Parameter & Ferkelfeeder & Rohrbreiautomat \\
\hline \multirow[t]{2}{*}{ Tier-Fressplatz-Verhältnis } & $1: 1$ & $5: 1$ \\
\hline & $\mathrm{x} \pm \mathrm{s}$ & $\mathrm{x} \pm \mathrm{s}$ \\
\hline Absetzmasse $(\mathrm{kg})(\mathrm{n}=128: 128)$ & $6,84 \pm 1,0$ & $6,86 \pm 1,0$ \\
\hline Ausstallmasse (kg) (n = $123: 123)$ & $25,6 \pm 4,5$ & $23,7 \pm 6,1$ \\
\hline Haltungstage & $45,4 \pm 3,4$ & $45,4 \pm 3,4$ \\
\hline Tägliche Zunahmen (g) (n = 123 : 123) & $411 \pm 78$ & $363 \pm 115 *$ \\
\hline \multicolumn{3}{|l|}{$* p<0,05$} \\
\hline \multicolumn{3}{|c|}{$\begin{array}{l}\text { Tabelle } 5 \\
\text { Variationskoeffizienten für die täglichen Zunahmen als Maß für das Auseinanderwachsen der F } \\
\text { Gruppen (\%) (Coefficients of variance for daily gain (\%) to measure the in-group heterogenity (\%)) }\end{array}$} \\
\hline Parameter & Ferkelfeeder/Tipp-Feeder & Rohrbreiautomat \\
\hline Tier-Fressplatz-Verhältnis & $1: 1$ & $5: 1 / 8: 1$ \\
\hline Ferkelfeeder : Breiautomat & 24,2 & 32,0 \\
\hline Tipp-Feeder : Breiautomat & 20,7 & 23,8 \\
\hline Ferkelfeeder : Breiautomat (Praxisbetrieb) & 18,9 & 31,8 \\
\hline
\end{tabular}

In der Zusammenfassung der 10 Durchgänge unter den Bedingungen der Forschungsstation traten kaum Unterschiede im täglichen Futterverbrauch (TierFressplatz-Verhältnis von $1: 1=0,73 \mathrm{~kg}$ Futter je Tier und Tag; Tier-FressplatzVerhältnis von $5: 1=0,70 \mathrm{~kg} /$ Tier und Tag) und in der Futterverwertung (Ferkelbzw. Tipp-Feeder: 1,64 kg Futter je kg Zuwachs; Rohrbreiautomat: ebenfalls 1,64 $\mathrm{kg} / \mathrm{kg}$ ) auf.

Im Praxisbetrieb wurden 256 gleichaltrige Ferkel so auf die beiden Gruppen in 4 Wiederholungen aufgeteilt, dass im Mittel die Lebendmasse nahezu identisch war (6,84 bzw. 6,86 kg, Tab. 4). Die Ferkel mit einem Tier-Fressplatz-Verhältnis von $1: 1$ 
erzielten auch unter diesen Bedingungen mit $411 \mathrm{~g}$ signifikant höhere tägliche Zunahmen bei gleichzeitig deutlich reduzierter Variabilität $(s= \pm 78 \mathrm{~g})$ im Vergleich zu Tieren, die bei einem Tier-Fressplatz-Verhältnis von $8: 1$ gefüttert wurden (363 \pm $115 \mathrm{~g})$.

In allen 14 Haltungsdurchgängen mit insgesamt 656 eingestallten Ferkeln entwickelten sich die Ferkel bei einem Tier-Fressplatz-Verhältnis von $1: 1$ ausgeglichener als am Breiautomaten (Tier-Fressplatz-Verhältnis $5: 1$ bzw. 8 : 1). Der Variationskoeffizient (als Maß für das Auseinanderwachsen der Tiere innerhalb der Gruppen) betrug bei einem Tier-Fressplatz-Verhältnis von bei $1: 1$ ca. 18,9 bis 24,2 \%, bei einem TierFressplatz-Verhältnis von 5 bis $8: 1$ dagegen 23,8 bis 32,0 \% (Tab. 5). Bei einer TierFressplatz-Relation von $8: 1$ war im Vergleich zu $1: 1$ der Unterschied zwischen den Variationskoeffizienten für die täglichen Zunahmen am größten.

\section{Diskussion}

Hinsichtlich des Tier-Fressplatz-Verhältnisses werden bei Verwendung von Breifutterautomaten in den Richtlinien 88 und 93/2001/EG sowie in der deutschen Tierschutz-Nutztierhaltungsverordnung keine Restriktionen vorgenommen. SCHÄFER (1999) untersuchte den Einfluss des Tier-Fressplatz-Verhältnisses ( $6: 1$ bis $10,5: 1)$ in Verbindung mit der Gruppengröße (12 bis 42 Tiere/Gruppe) auf die täglichen Zunahmen und den Variationskoeffizienten für die Zunahmen bei Absetzferkeln. Sie wies nach, dass mit steigender Gruppengröße und weiter werdendem TierFressplatz-Verhältnis die Zunahmen absanken und der Variationskoeffizient größer wurde, konnte versuchstechnisch die Effekte von Gruppengröße und Tier-FressplatzRelation jedoch nicht voneinander trennen. Nach KIRCHER et al. (2000) ist ein TierFressplatz-Verhältnis von $10: 1$ im Vergleich zu 6,7 : 1 kritisch zu bewerten, da tendenziell mehr Verdrängungen am Trog und geringere tägliche Zunahmen bei den Absetzferkeln auftreten. Trotz dieser kritischen Stimmen gibt es einen Trend hin zu größeren Gruppen und weiter werdendem Tier-Fressplatz-Verhältnis (HOY, 2004, 2005), da eine Kostendegression pro Ferkel(platz) angestrebt wird. Dabei werden jedoch ethologische Gegebenheiten und die Auswirkungen auf Gesundheit und Leistung missachtet. So existiert beim Schwein ein typischer biphasischer Rhythmus der Futteraufnahme mit einem Peak am Vormittag und einem deutlich stärker ausgeprägten Anstieg in den späten Nachmittagsstunden (SCHÄFER und HOY, 1997; SCHÄFER, 1999; LEXER et al., 2000; HOY et al., 2001). Dieses arttypische Futteraufnahmeverhalten kollidiert mit einem eingeschränkten Tier-FressplatzVerhältnis, selbst wenn eine bestimmte Plastizität im Verhalten der Tiere unterstellt werden kann. Die eigenen Untersuchungen sowohl unter den vergleichsweise gut zu standardisierenden Haltungsbedingungen einer Forschungsstation wie auch unter Feldbedingungen ergaben übereinstimmend, dass die täglichen Zunahmen bei einem Tier-Fressplatz-Verhältnis von $1: 1$ stets höher (z.T. statistisch abgesichert) als bei einem weiteren Verhältnis von 5 bis 8 Ferkeln pro Fressplatz waren. Die Überlegenheit betrug zwischen $25 \mathrm{~g}$ und $48 \mathrm{~g}$ höhere Tageszunahmen zugunsten der Fütterungstechnik mit einem Fressplatz pro Tier, was einem Leistungsvorteil zwischen 5,8 \% und 13,2 \% entspricht. Die Niveau-Unterschiede in den täglichen Zunahmen zwischen Forschungsstation und Praxisbetrieb bzw. zwischen den Durchgängen mit Ferkelfeeder und Tipp-Feeder sind zum einen durch Unterschiede im Absetzalter (im Mittel zwischen 21 und 28 Tagen), unterschiedliche Genetik und Haltungsbe- 
dingungen (Forschungsstation vs Praxisbetrieb) sowie z.T. auf den Einsatz medikamentierten Futters nach Auftreten von Durchfällen zu erklären. Zugleich entwickelten sich die Tiergruppen bei einer Tier-Fressplatz-Relation von $1: 1$ deutlich homogener, was in dem um 3,1 \% bis 12,9 \% niedrigeren Variationskoeffizienten für die täglichen Zunahmen am Ferkel- bzw. Tipp-Feeder gegenüber dem Rohrbreiautomaten zum Ausdruck kommt. Umgekehrt ist zu konstatieren, dass die Tiere mit weitem TierFressplatz-Verhältnis deutlich stärker auseinander wuchsen als die Vergleichstiere in Gruppen, bei denen jedes Tier einen Fressplatz hatte. Die biologische Begründung für die homogenere Entwicklung der Tiere bei einem Tier-Fressplatz-Verhältnis von 1:1 wird in der größeren Chancengleichheit bei dem Fütterungssystem mit Platz für das gleichzeitige Fressen aller Ferkel der Gruppe gesehen. Somit kann nicht nur die Gruppengröße eine Erhöhung der Varianz für die Lebendmassezunahme innerhalb der Gruppe bewirken (WOLTER et al., 2000; KIRCHER et al., 2000), sondern auch ein (deutlich) erweitertes Tier-Fressplatz-Verhältnis. Die Fressplatzbreite bzw. das Verhältnis von Tieren zu Fressplätzen wird kontrovers diskutiert. Idealerweise sollten nach MADEC et al. (2003) Buchten so mit Fressplätzen ausgestattet sein, dass mindestens die Hälfte der Ferkel zu jeder Zeit gleichzeitig fressen kann, was einem Tier-Fressplatz-Verhältnis von 2 : 1 entspricht. BAXTER (1989) erarbeitete eine Formel $\left(\mathrm{W}=61 \times \mathrm{BW}^{0,33}\right)$ mit $\mathrm{W}=$ Fressplatzbreite und BW = Lebendmasse, nach der für ein $10 \mathrm{~kg}$ schweres Schwein eine Fressplatzbreite von $13 \mathrm{~cm}$ vorhanden sein sollte. Es ist möglich, dass bei Optimierung aller Haltungsbedingungen auch bei einer größeren Zahl an Schweinen pro Fressstelle hohe Leistungen und eine gute Tiergesundheit erreichbar sind, wenngleich der Einfluss der Rangordnung auf das Futteraufnahmeverhalten und die Leistung unter diesen Bedingungen bereits seit längerem bekannt ist (PUPPE et al., 1991) und kürzlich erneut nachgewiesen wurde (SCHÖNFELDER, 2005). Allerdings darf nicht übersehen werden, dass in den letzten Jahren im Zusammenhang mit steigenden Bestandsgrößen auch die tiergesundheitlichen Probleme bei Absetzferkeln größer geworden sind (dargestellt am Beispiel der USA - OHLINGER, 2003). Sowohl die Anzahl der klinischen Erkrankungen nach Infektion mit dem PRRS- und/oder porcinen Circovirus als auch nach StreptokokkenInfektion ist danach in den USA deutlich angestiegen und nach dem Verbot der antibiotischen Leistungsförderer muss auch in Europa mit einer Zunahme der Krankheitsfälle gerechnet werden (HOY und von BORELL, 2002). Umso wichtiger ist es, über eine tiergerechte Fütterungstechnik keine zusätzliche Belastung für die Tiere zu schaffen und zumindest im absetznahen Zeitraum eine weitgehend ungestörte Futteraufnahme für alle Tiere der Gruppe zeitlich synchron entsprechend dem arttypischen Futteraufnahmeverhalten zu ermöglichen. Ein Tier-Fressplatz-Verhältnis von (annähernd) $1: 1$, das mit neuen Fütterungstechniken (Ferkelfeeder, Tipp-Feeder), aber auch mit Ende der 90er Jahre entwickelten Längstrog-Intervallfütterungssystemen verschiedener Hersteller (HOY, 2001) realisiert werden kann, unterstützt den Übergang von der Ernährung an der Sau hin zu einer technischen Fütterung der Absetzferkel. Die bisherigen Ergebnisse zur Tiergesundheit zeigen an, dass die Durchfallmorbidität bei einer rationierten Fütterung im absetznahen Zeitraum bei einem TierFressplatz-Verhältnis von 1 : 1 deutlich geringer sein kann als bei Sattfütterung und einer Tier-Fressplatz-Relation von 5 bis $8: 1$ (KNOOP et al., 2005).

Zusammenfassend ist festzustellen, dass Rohrbreiautomaten kostengünstig und technisch einfach aufgebaut sind. Allerdings muss das Tier-Fressplatz-Verhältnis bei 
der Sattfütterung der Absetzferkel beherrscht werden. Es ist dringend zu empfehlen, in der absetznahen Phase (etwa bis zwei Wochen nach dem Absetzen) zusätzliche Ferkeltröge in die Buchten zu stellen, um ein annäherndes Verhältnis von Ferkeln zu Fressplätzen von $1: 1$ zu erreichen. Vorzüglich sind neue technische Entwicklungen mit einem Tier-Fressplatz-Verhältnis von 1:1 zumindest im absetznahen Zeitraum. Ein enges Tier-Fressplatz-Verhältnis verringert die Belastung bei der Futteraufnahme vor allem für die kleineren Absetzferkel und gewährleistet bei höheren Zunahmen (im Vergleich zu Techniken mit Tier-Fressplatz-Verhältnissen von 5 bis $8: 1$ oder mehr) eine gleichmäßigere Entwicklung aller Ferkel der Gruppe.

\section{BAXTER, S.H.:}

\section{Literatur}

Designing the pig pen. In: BARNETT, J.L.; HENESSY, D.P.: Manipulating Pig Production II. Australasian Pig Science Association, Werribee (1989), 191-206

BROOKS, P.H.; TSOURGIANNIS, C.A.:

Factors affecting the voluntary feed intake of the weaned pig. In: PLUSKE, J.R.; LE DIVIDICH, J.; VERSTEGEN, M.W.A.: Weaning the pig. Concepts and consequences. Wageningen Academic Publisher (2003), 81-116

DONE, S.:

Enteritic and respiratory diseases in the young weaned pig. In: VARLEY, M.A.; WISEMAN, J.: The Weaner Pig, Nutrition and Management. CABI Publishing (2001), 223-248

HELD, S.; MENDL, M.:

Behaviour of the young weaner pig. In: VARLEY, M.A.; WISEMAN, J.: The Weaner Pig, Nutrition and Management. CABI Publishing (2001), 273-297

HOY, ST.:

Haltung und Fütterung von Absetzferkeln - Die Gesundheit steht im Vordergrund. DGS Magazin (2001) 5, 40-42

HOY, ST.:

Neuheiten auf der EuroTier 2004 im Bereich Schweinehaltung. Neue Landwirtschaft (2004) 12, 54-56

HOY, ST.: HOY, ST.:

Haltung und Fütterung von Absetzferkeln. DGS Magazin (2005) 50, 35-38

Der Tipp-Feeder - ein neues Fütterungssystem für Absetzferkel. Landtechnik 61 (2006) 4, 216-217

HOY, ST.; MEHLHORN, G.:

Zum postnatalen Verhalten der Ferkel unter besonderer Berücksichtigung der Herausbildung der Saugordnung und ihre Beziehung zur Lebendmasseentwicklung. Mh. Vet. Med. 44 (1989), 16-20

HOY, ST.; PUPPE, B.:

Effects of teat order on performance and health in growing pigs. Pig News and Informations 13 (1992)

3, 131-136

HOY, ST.; BORELL, E. v.:

Beitrag der Tierhaltung zur Sicherheit tierischer Produkte. Züchtungskunde 74 (2002) 6, 430-437

HOY, ST.; ZIRON, M.; LEONHARD, P.; KINGSLEY OPPONG SEFA:

Untersuchungen zum Futteraufnahmeverhalten ad libitum gefütterter tragender Sauen in Gruppenhaltung am Rohrautomaten. Arch. Tierz., Dummerstorf 44 (2001) 6, 629-638

KIRCHER, A.; WEBER, R.; WECHSLER, B.; JUNGBLUTH, T.:

Verhalten und Leistung von Aufzuchtferkeln an Rohrbreiautomaten bei unterschiedlichem Tier-Fressplatz-Verhältnis. Proc. Aktuelle Arbeiten zur artgemäßen Tierhaltung, KTBL-Schrift 403 (2000), 120127

KNOOP, ST.; HOY, ST.:

Ein neues Fütterungssystem für Absetzferkel - der Ferkelfeeder. Proc. 7. Tagung Bau, Technik und Umwelt. Braunschweig 1.-3.3.2005, 193-198

KNOOP, ST.; HOY, ST.; MOLL, H.-R.:

Ferkelfeeder - ein neues Fütterungssystem für Absetzferkel. Landtechnik 60 (2005) 3, 166-167

LEXER, D.; BAUMGARTNER, J.; TROXLER, J.:

Einfluss von Gruppengröße und Gruppenzusammensetzung auf die Tagesperiodik des Verhaltens von Absetzferkeln. Proc. Aktuelle Arbeiten zur artgemäßen Tierhaltung, KTBL-Schrift 403 (2000), 46-53 
MADEC, F.; LE DIVIDICH, J.; PLUSKE, J.R.; VERSTEGEN, M.W.A.:

Environmental requirements and housing of the weaned pig. In: PLUSKE, J.R.; LE DIVIDICH, J.; VERSTEGEN, M.W.A.: Weaning the pig. Concepts and consequences. Wageningen Academic Publisher (2003), 337-360

MORMEDE, P.; HAY, M.:

Behavioural changes and adaptations associated with weaning. In: PLUSKE, J.R.; LE DIVIDICH, J.; VERSTEGEN, M.W.A.: Weaning the pig. Concepts and consequences. Wageningen Academic Publisher (2003), 53-60

OHLINGER, V.F.:

Viruserkrankungen beherrschen - PRRS, Circovirusinfektionen und andere. Stand der Dinge und Möglichkeiten der Bekämpfung und Vorbeugung. Proc. AVA-Fachtagung 19./20. Februar 2003.

PUPPE, B.; HOY, ST.; JAKOB, M.; WULLBRANDT, H.:

Erste Ergebnisse zur Sozialordnung weiblicher und männlicher Mastschweine bei gemischter und geschlechtergetrennter Haltung in Beziehung zur Lebendmasseentwicklung und ausgewählten Verhaltensweisen. Mh. Vet. Med. 46 (1991), 515-519

PUPPE, B.; TUCHSCHERER, M.; HOY, ST.; TUCHSCHERER, A.:

Soziale Organisationsstrukturen beim intensiv gehaltenen Schwein. 1. Ethologische Untersuchungen zur Saugordnung. Arch. Tierz., Dummerstorf 36 (1993) 5, 539-550

SCHÄFER, E.:

Vergleichende Untersuchungen des Nahrungsaufnahmeverhaltens und der Wachstumsintensität von Mastschweinen und Ferkeln an Rohrbreiautomaten und anderen Fütterungssystemen unter besonderer Berücksichtigung der Gruppengröße. Diss. Univ. Gießen 1999

SCHÄFER, E.; HOY, ST.:

Wie viele Schweine an einem Rohrbreiautomaten? Schweinezucht und Schweinemast 45 (1997) 5, 2224

SCHÖNFELDER, A.:

The effect of rank order on feed intake and growth of fattening pigs. Dtsch. Tierärztl. Wschr. 112 (2005), 215-218

WOLTER, B.F.; ELLIS, M.; CURTIS, S.E.; PARR, E.N.; WEBEL, D.M.:

Group size and floor-space allowance can affect weanling-pig performance. J. Anim. Sci. 78 (2000), 2062-2067

Eingegangen: 08.08.2006

Akzeptiert: 15.12.2006

Anschrift der Verfasser

Prof. Dr. STEFFEN HOY*, Dipl.-Ing. agr. STEPHANIE KNOOP, CARMEN WEIRICH

Institut für Tierzucht und Haustiergenetik

Justus-Liebig-Universität Gießen

Bismarckstr. 16

35390 GIESSEN

*Autor für Korrespondenz, E-Mail: Steffen.Hoy@agrar.uni-giessen.de 